
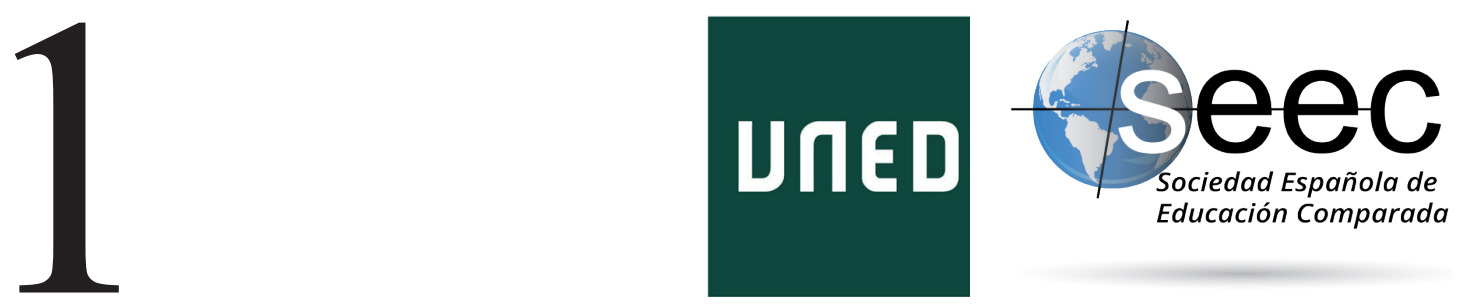

\title{
Editorial: La educación en los países emergentes: ¿nuevos laboratorios educativos del siglo XXI?
}

Editorial: The education in the emergent countries: new education laboratories of the XXIst century?

\section{Ana Ancheta-Arrabal*}

DOI: $10.5944 /$ reec.39.2021.31001

\author{
Recibido: 7 de junio de 2021 \\ Aceptado: 7 de junio de 2021
}

\footnotetext{
*Ana Ancheta-Arrabal: Profesora Contratada Doctora en el Departamento de Historia y Educación Comparada de la Facultad de Filosofía y Ciencias de la Educación de la Universidad de Valencia trabaja las líneas de investigación centradas en las políticas y sistemas de Educación y Atención de la Primera Infancia, el derecho a la educación y la equidad educativa desde la perspectiva y el ámbito de la educación comparada e internacional. Datos de contacto: E-mail: ana.ancheta@uv.es. ORCID: https://orcid.org/oooo-0oo29833-242X y Scopus AutHor ID: 57201408839
} 
La denominación de mercados o economías emergentes ha categorizado de forma fluida a determinadas naciones bajo la designación de un fenómeno económico o comercial desde la última década del pasado siglo (Authers, 2006). Entre estos países, los denominados como BRICS (Brasil, Rusia, India, China y Sudáfrica) se consideran un grupo económico importante, dada su enorme expansión económica en la última década y, al menos en algunos casos, por su transición desde la relativa desaceleración económica hacia un crecimiento económico dinámico (Industry Week, 2011; Follath \& Hesse, 2014).

Frente al intenso debate que la emergencia de estas economías ha generado para identificar el papel que desenvuelven como conjunto en el marco de las relaciones internacionales, el grupo BRICS ha atendido a la advertencia de transitar gradualmente de la convergencia hacia una cierta normatividad con respecto a temáticas claves para su permanencia y expansión en el horizonte de una mejor adaptación al sistema multilateral en formación, aumentando la coherencia del grupo exponencialmente e inclusive abordando áreas de cooperación poco conocidas que incluyen sectores como ciencia, agricultura, creación de un Think Tank, entre otros, lo que le ha promovido más allá de una realidad transitoria en el plano internacional (Oliver Stuenkel, 2017). Aunque las declaraciones efectuadas en las cumbres de este grupo se centran en la cooperación económica y comercial mutua (BRICS Think Tanks Council, 2013), también ha habido intentos considerables de mejorar la colaboración en la educación, así como en la investigación por las enormes oportunidades que pueden contribuir a su consolidación económica frente a los diversos desafíos que comparten estos países (Havlik et. al., 2009; Rensburg, et. al., 2015). Representando un hito tanto en la asociación BRICS como en la colaboración de la UNESCO con el Grupo en cuestión, en la consulta organizada en el marco de la $37^{\mathrm{a}}$ Sesión de la UNESCO Conferencia general en París en noviembre de 2013 los Ministros de educación acordaron unir fuerzas junto con la UNESCO para apoyar el progreso de la educación en el nivel mundial a través de acciones coordinadas. Igualmente, se instaba a trabajar en la promoción y establecimiento de un mecanismo para la cooperación permanente en educación, invitando a este organismo internacional a desempeñar un papel central en el apoyo de su acción colectiva. Desde entonces, el compromiso del Grupo con la educación y la investigación en las cumbres BRICS celebradas ha puesto de manifiesto los intentos para sentar las bases de esta acción colectiva afirmando la importancia estratégica de la educación para el desarrollo sostenible y el crecimiento económico inclusivo y comprometido para fortalecer la cooperación.

Estos países han aumentado su gran potencial inicial para influir individualmente en las tendencias educativas mundiales a través de la experiencia que pueden compartir, de sus roles crecientes como socios de desarrollo y de los nuevos enfoques que está desarrollando para la cooperación internacional. Como resultado durante los últimos años, se ha transformado el mapa mundial de la educación, escolarizando millones de estudiantes, estableciendo centros de aprendizaje de clase mundial, que impulsan la innovación y comparten experiencia y conocimiento (UNESCO, 2014).

Desde este escenario, se abrió la llamada del número que aquí se presenta en torno al estudio de los avances, tendencias y desafíos de la educación y la investigación comparada en este grupo de países con la finalidad de contribuir tanto al objeto de su conocimiento como a identificar las potencialidades del mismo para la aplicación científica y académica del público especializado y para su interés del público general. A tales efectos, la primera de las contribuciones a cargo del profesor Wolhuter-Emerging Countries as Taxon in Comparative and International Education- explora las posibilidades como unidades 
supranacionales de comparación tanto del taxón de los países emergentes como, dentro de los mimos, del denominado grupo BRICS en el ámbito de la Educación Comparada y la Educación Internacional. Como el mismo autor indica, su trabajo de trayectoria previa viene motivado desde el intento de superar la predilección por el Estado nación como unidad de análisis predominante entre comparatistas, pero también sin olvidar que en sí misma también constituye una fuerza poderosa en la configuración de los sistemas educativos nacionales que, a su vez, siguen integrando una característica potente e indisoluble del escenario global. En todo caso, también justifica esta necesidad de pasar del nivel de análisis nacional al supranacional en particular desde la propia motivación por la que la revolución tecnológica internacional de comunicaciones y transporte y la economía internacional resultante ineludiblemente unida a la globalización y a la movilidad demográfica, junto con la crisis ecológica y otros desafíos globales como el terrorismo, han despojado simplemente tanto de la impermeabilidad (real o retratada) de las fronteras nacionales y la soberanía nacional como de valor a este nivel de análisis en la Educación Comparada (autor citando a Kamens, 2017), que se suma al surgimiento de estructuras globales desde las que dichas agrupaciones supranacionales también han comenzado a tener más sentido. A partir de aquí, el autor ofrece un estudio del grupo de países emergentes, como posible taxón para los estudios en la educación comparada e internacional, sobre los contextos sociales y el desarrollo educativo de estos países con el objetivo de determinar la homogeneidad interna y la varianza entre ellos. A pesar de no representar una categoría demasiado homogénea, se concluye que en sí mismo este grupo de trayectorias educativas a la vanguardia de las naciones del Sur Global y en la orientación del desarrollo educativo, representa un laboratorio educativo para los estudios de la Educación Comparada e Internacional en el que sugiere utilizar el tamaño y la calidad del esfuerzo educativo y la fuerza de la economía como criterios de comparación significativos, ambos explorados en estudios precedentes sobre estos países (Van Damme, 2017).

Una buena muestra de esta apuesta por aplicar las unidades supranacionales en lo estudios comparativos de las políticas educativas en los países emergentes es la contribución de las profesoras Oliveira-Dri y Pulido-Montes que lleva por título «Los Planes Educacionales del MERCOSUR y la recontextualización de sus políticas en Brasil», en línea de otros estudios comparativos que analizan el papel que desempeñan determinadas normas e instituciones en la defensa de los órdenes mundiales e investigan su relación con el surgimiento de nuevos centros de poder, en particular del grupo de países emergentes que pertenecen al BRICS (Leal Rinaldi y Soreanau Pecequilo, 2021). En lo referente a las convergencias en el ámbito educativo, se plantea la perspectiva entre los miembros de los BRICS teniendo como parámetro de referencia el Proceso Europeo de Bolonia, que supone un caso paradigmático en el fortalecimiento de la cooperación en la perspectiva educativa y de investigación (de Araújo Borges, 2018, p. 48). Desde este enfoque, el estudio planteado por estas autoras parte del contexto de procesos de regionalización que han generado sinergias y confluencias que permiten desarrollar economías y bloques más competitivos en el que surge el Mercado Común del Sur (MERCOSUR) en la región Sur de América Latina para centrarse en el proceso de construcción del Sector Educativo del MERCOSUR (SEM) como caso particular de la conformación de una agenda política educativa compartida por los países que integran el bloque regional Sudamericano y, de modo más específico, del escenario emergente que supone Brasil que representa un actor principal en la disputa por la hegemonía de la región desde el comienzo de la fundación del bloque regional (autoras citando a Granato, 2020). El 
análisis sincrónico planteado de los Planes del SEM revela las evoluciones y continuidades que han guiado las políticas que se han desarrollado desde el MERCOSUR como son aquellas de acreditación, movilidad, formación del profesorado, financiación, calidad y la educación como elemento de integración, y donde las políticas de acreditación, movilidad y de la Educación Superior se establecen como catalizadoras de la integración de la región, implicando una mayor prioridad en su agenda educativa.

No en vano, el conocimiento de procesos de regionalización como el anterior permite la comprensión del funcionamiento de nuevos sistemas de orden internacional como conjunto de principios, reglas, procedimientos y instituciones, aceptadas por los actores principales -en este caso, Estados- que establecen una interdependencia permanente y un marco de negociación de sus respectivas metas y expectativas, con el objetivo de defender intereses comunes y que, como cualquier otro tipo de orden, les obliga a cumplir con las normas legales. Igualmente, para que perdure, debe alcanzar cierto grado de legitimidad, ya que necesita continuamente resaltar sus beneficios y por lo tanto fortalecer su superioridad frente a otras alternativas disponibles. Este tipo de desafío puede conducir gradualmente a modificaciones de la propia naturaleza de la norma, mientras que el discurso justificativo se ocupa del claro problema de determinar cuál de los actores apoyará las normas para un tema en particular. La pregunta crucial, en este caso, gira en tono a aquellas normas en las que se produce el acuerdo que, en principio, puede ser autorizado. Desde el plano normativo, el análisis presentado por el profesor Guillermo Ruíz, «El derecho a la educación: definiciones constitucionales comparadas en América del Sur» ofrece respuestas a dicho interrogante mediante el análisis comparativo de las cláusulas constitucionales sobre el derecho a la educación en cuatro países que integran o están asociados al MERCOSUR (Argentina, Brasil, Uruguay como países miembros y Chile como Estado asociado), desentrañando la influencia que entidades supranacionales como esta ocupan como laboratorio normativo en los procesos de armonización normativos que posibilitan las reformas políticas en materia de educación debido a la profusión de claúsulas constitucionales sobre la educación como derecho en estos países emergentes. Para este autor, a pesar de lo ambiciosas que puedan parecer dichas disposiciones, constituyen una importante apuesta para avanzar en el reconocimiento y en la protección de los derechos individuales y colectivos, y así favorecer un acceso a la justicia más extendido, una mayor integración social y la promoción de un sistema judicial tanto más permeable a las demandas sociales como que posibilite que las decisiones judiciales puedan influir en la implementación de los programas gubernamentales.

A este respecto, el derecho a la educación en las potencias emergentes converge entre los principales desafíos para la mejora de la agenda de cooperación entre estos países y, en especial, del grupo de los BRICS que se viene enfrentando a la persistencia de las demandas encaminadas a implementar la agenda de Educación para Todos (EPT) (de Araújo Borges, 2018). Objeto central de la siguiente de las contribuciones del monográfico a cargo de Liya Kalinnikova Magnusson que aborda un estudio de caso de la Federación de Rusia en relación con las reformas normativas motivadas por el movimiento de la EPT. Una vez más, el enfoque hermeneútico sustenta la base del planteamiento sobre la descripción e interpretación de recopilaciones normativas que la autora analiza mediante la investigación histórica y fenomenológica, para responder al propio enunciado de su propuesta Education laboratories in <education for all in Russia: from Lenin to Putin L. Como resultado del análisis planteado, es posible determinar cómo las declaraciones ideológicas formuladas en las primeras Constituciones del país desempeñaron un papel 
crucial en la definición de la formación estructural y el derecho a la educación, incluyendo a la infancia con discapacidad; abriendo el debate desde el supuesto principal de que la educación especial dentro de dichos períodos de desafíos fundamentales del país reactiva sus orígenes e interconecta con un contexto político más amplio hacia soluciones para hacer posible la educación para todos en la Federación de Rusia.

En este último sentido, los avances en la cooperación internacional han demostrado que, así como en los países desarrollados, en los BRICS la internacionalización ha tenido un efecto positivo para la innovación y evidencian la necesidad de utilizar como estrategia la expansión internacional como herramienta para ampliar su capacidad de inversión (Bansi et. al., 2021). Una tendencia que bien puede ser extrapolable al ámbito de la cooperación multilateral en educación superior entre los países de las denominadas cinco mayores enonomías emergentes, tal y como se desprende de la investigación realizada por Jin Sun y Aiyi Yang a propósito de la llamada de este número especial de la revista Optimism Beyond the Problems: BRICS Higher Education Cooperation from a Chinese Perspective. Partiendo de las evidencias que trabajos previos han demostrado en este ámbito y del interés que ha suscitado para el debate científico y académico de la Educación Comparada, estos autores identifican los logros principales de esta cooperación, destacando el establecimiento de dos proyectos (la Universidad de Red de BRICS y la Liga de Universidades de BRICS) y el desarrollo de una serie de acciones en el intercambio de estudiantes y en la investigación científica, así como los problemas existentes como las dificultades de cooperación multilateral y de confirmación mutua de créditos y calificaciones, y el entusiasmo insuficiente entre los posibles participantes. Si bien la cooperación en educación superior de estos países ha compartido la «Solución BRICS» con el mundo y las valiosas experiencias prácticas adquiridas se pueden aplicar al desarrollo de la educación superior en el Sur Global (Li, 2018 citado por los autores), las problemáticas redundan en la cooperación internacional mostrando cómo la falta de recursos y la mejora de la colaboración pone en riesgo este tipo de empresas (Bansi et. al, 20121). Con todo, las opiniones positivas y optimistas de los expertos chinos contrastan con los problemas, obstáculos y expectativas pesimistas dominantes de estudios anteriores, lo que indica que la cooperación en la educación superior entre los países BRICS no solo cuenta con el apoyo del gobierno chino, pero también con la aceptación de los participantes chinos como premisa para su sostenibilidad futura.

Estudios como el anterior, claramente manifiestan la relevancia significativa que ha cobrado la cooperación en educación superior entre los países BRICS y sus posibles beneficios como brindar a estudiantes y docentes más oportunidades de intercambio y cooperación internacional, mejorar tanto la internacionalización y la cooperación internacional como la competitividad de las universidades, o promover intercambios culturales y los lazos entre pueblos entre dichos países. Pero también cómo el papel de los países BRICS en la gobernanza educativa global está progresando y creando un modelo de cooperación en educación superior que difiere del modelo de cooperación de los países del Norte Global y que es más aplicable a los países del Sur Global. Como ejemplo inspirador de esta mejora, la profesora Takako Mino explora en su aportación Building a Liberal Arts Tradition in India, las nuevas universidades de artes liberales en la India como experiencia que está adoptando enfoques innovadores y haciéndolos propios a la idiosincrasia de este país, a medida que cambian las demandas de los graduados universitarios en un mundo altamente complejo e interconectado. De esta indagación se desprende que el modo en que la India aborda las reformas educativas y las ajusta a su 
propia cultura puede potencialmente servir como un modelo a tener en cuenta para que los mercados emergentes puedan actualizar desde el ámbito de la educación superior el desarrollo sostenible centrado en el ser humano basado en sus fortalezas y necesidades únicas. Una visión que algunos investigadores han señalado sobre los países BRICS por la que se oponen a la visión estrecha del capitalismo humano, reafirmando el papel no económico de la educación en el desarrollo, al subrayar en su objetivo general de cooperación educativa BRICS el de fomentar los «intercambios culturales» a través de «intercambios de personas a personas» (Muhr y Azevedo, 2018).

Esta visión se evidencia a través de las experiencias de aprendizaje y servicio desarrolladas en todo el mundo, pero especialmente en países emergentes y en el ámbito de la educación superior tal y como da cuenta el estudio presentado por Rocío López García-Torres, Elia Saneleuterio Temporal y Nuria Andreu Ato «De la formación docente en España a la práctica educativa en Senegal: un proyecto de cooperación internacional basado en el aprendizaje servicio para Infantil y Primaria» y manifiesta para los intereses de la educación para la justicia global. En el nivel nacional, la cooperación se considera un complemento importante de la diplomacia pública del país, que ayuda a promover los intercambios culturales y los vínculos entre pueblos de los países BRICS y como experiencia consigue vincular teoría y práctica con unos beneficios considerables entre los que destaca el de la conciencia sobre el valor fundamental de la educación como instrumento para combatir la pobreza y la exclusión social.

El irrenunciable papel que la educación desempeña en el desarrollo sostenible y a través de compromisos internacionales como la Agenda 2030 pone de relieve, de nuevo, la importancia de atender a sinergias como las que propician los proyectos oficiales de enseñanza de lenguas extranjeras y de difusión cultural en países emergentes, como es el caso de la última contribución al monográfico que presenta Amparo Rodrigo-Andreu. Bajo el título «La cooperación educativa y cultural entre España e India. Logros y retos» la autora visibiliza la necesidad de cooperación y de coordinación entre las diferentes instituciones involucradas en la enseñanza del idioma Español en la India a través del análisis de la situación actual y de las posibilidades de maximizar la cooperación bilateral en materia educativa y cultural entre España e India.

Algunos desafíos para el progreso de la agenda de cooperación entre las potencias emergentes de los BRICS en el marco de la elaboración de alianzas entre el MERCOSUR y otros bloques o alianzas supranacionales pueden indicar una tendencia de marginación de los BRICS en las estrategias regionales y la asunción de centralidad del MERCOSUR y su agenda (de Araújo Borges, 2018). Con todo, los temas clave de este proceso constituyen una agenda común y relevante para la educación superior en el escenario global y regional, abordando temas más allá de las fronteras nacionales, así como enfrentando los desafíos que el grupo BRICS y resto de países emergentes tienen planteados en lo que respecta a la reducción de las desigualdades educativas. Como se ha puesto de manifiesto, la consolidación de la posición de los países BRICS en los escenarios global y regional depende de esta articulación entre las agendas globales e internacionales de excelencia en la educación superior y los históricos retos relacionados con el derecho a la educación y su democratización, que siguen pendientes de implementación en un contexto de crisis global de la educación si cabe aún más desafiante que la actual pandemia y crisis sanitaria han impuesto.

Tanto es así que el pasado 1 de Junio de 2021 los ministros de exteriores del grupo BRICS expresaban en una reunión su Joint Statement on Stregthening and Reforming 
de Multilateral System la necesidad de reformar el sistema multilateral en tiempos de pandemia y crisis mundial desde la base, en otros aspectos, de tornar los instrumentos de la gobernanza global más inclusivos, representativos y participativos, así como sintonizados con las realidades contemporáneas en una participación más significativa de los países en desarrollo y menos desarrollados; señalando la evolución del modelo de compromiso propio del grupo basado en el consenso y el esfuerzo colectivos. El seguimiento de estas y otras propuestas como de las iniciativas conjuntas derivadas del establecimiento del grupo BRICS o de otras alianzas y consensos entre los llamados países emergentes en el terreno de la educación, sin duda prometen una fuente de análisis interesante mientras sigan en el punto de mira internacional, no sólo por un interés actual desde el Sur Global pero también dinámico hacia el Norte Global en sus trascendencias.

\section{Referencias}

Authers, J. (2006). "The Long View: How adventurous are emerging markets?" Published: October 20, 2006. The Financial Times. Disponible en; http://www. ft.com/cmc/s/be77e6oo. 4

Bansi,A.C.; deAndrade, A.M. F.y Galina, S.V.(2021)A relação entre rnternacionalizaçãoe inovação nas multinacionais de países emergentes. Revista Eletrônica de Negócios Internacionais: Internext, 16(1), pp. 110-126

BRICS Think Tanks Council. (2013). Declaration on the Establishment of the BRICS Think Tanks Council. South Africa: Durban.

de Araújo Borges, M.C. (2018). BRICS e a Educação Superior: Questões e Convergências Possíveis? Revista Ibero-americana de Educação, 76(1) [(2018/04/o1), pp. 39-56

Follath, E., y Hesse, M. (2014). Troubled Times: Developing Economies Hit a BRICS wall. Disponible en http://www.spiegel.de/interna tional/world/economy-slowsin-brics-countries-as-worries-mount-a-951453.html

Havlik, P., R. Stollinger, O. Pindyuk, G. Hunya, B. Dachs, C. Lennon, M. P. Ribeiro, J. Ghosh, W. Urban, V. Astrov, y E. Christie. (2009). EU and BRICs: Challenges and opportunities for European competitiveness and cooperation. Brussels: European Commission.

Industry Week. (2011). BRIC Countries Face Both Growth and Crucial Challenges. Disponible en http://www.industryweek.com/articles/bric_countries_face_ both_growth_and_crucial_challenges_25282.aspx

Kamens, D.H. (2017). Beyond the Nation State: The reconstruction of nationhood and citizenship. Bingley: Emerald.

Leal Rinaldi, A. y Soreanau Pecequilo, C. (2021). The Contemporary World Order, BRICS, and the R2P Principle: the Cases of Brazil and China (2005/2017). Colombia Internacional, 105, pp. 3-28. DOI: https://doi.org/10.7440/ colombiaint105.2021.01 
Li, Y. (2018). Development of Cooperation in Higher Education in BRICS Countries. Changing Societies \& Personalities, 2(4), pp. 393-405. DOI:10.15826/ csp.2018.2.4.053

Ministry of external affairs, Government of India (2021). BRICS Joint Statement on Strengthening and Reforming the Multilateral System. Disponible en: https:// mea.gov.in/bilateral-documents.htm?dtl/33888/BRICS_Joint_Statement_on_ Strengthening_and_Reforming_the_Multilateral_System

Muhr, T., y Azevedo, M. D. (2018). The BRICS development and education cooperation agenda. Vestnik RUDN. International Relations, 18(3), pp. 517-534. DOI: 10.22363/2313-0660-2018-18-3-517-534

Rensburg, I., Motala, S. y Arulraj David, S. (2015). Opportunities and challenges for research collaboration among the BRICS nations. Compare: A Journal of Comparative and International Education, 45(5), pp. 814-818, DOI: 10.1080/03057925.2015.1074823

Stuenkel, O. (2017). BRICS, e o futuro da ordem global. Rio de Janeiro - São Paulo: Paz \&Terra.

United Nations, Educational, Scientific and Cultural Organization (UNESCO). (2014). BRICS Building Education for the Future. UNESCO. Disponible en: https:// unesdoc.unesco.org/ark:/48223/pfoooo229692

Van Damme, D. (2017) Have emerging Latin American countries chosen quantity over quality in education? OECD Education and Skills Today, March, 31, 2017. Disponible en: https://oecdedutoday.com/have-emerging-latin-american-countries-chosenquantity-over-quality-in-education/ 